\title{
A LOWER BOUND FOR THE DIMENSION OF A HIGHEST WEIGHT MODULE
}

\author{
DANIEL GOLDSTEIN, ROBERT GURALNICK, AND RICHARD STONG
}

\begin{abstract}
For each integer $t>0$ and each simple Lie algebra $\mathfrak{g}$, we determine the least dimension of an irreducible highest weight representation of $\mathfrak{g}$ whose highest weight has width $t$. As a consequence, we classify all irreducible modules whose dimension equals a product of two primes. This consequence, which was in fact the driving force behind our paper, answers a question of N. Katz.
\end{abstract}

\section{INTRODUCTION}

Throughout this paper $\mathfrak{g}$ is a simple Lie algebra of rank $n$ over the complex numbers $\mathbb{C}$. The irreducible finite-dimensional $\mathfrak{g}$-modules are the highest weight modules $V(\omega)$ where $\omega$ is a dominant weight. Each $\omega$ is uniquely a linear combination $\omega=\sum_{1 \leq i \leq n} a_{i} \omega_{i}$ of the fundamental dominant weights $\omega_{i}, 1 \leq i \leq n$, where the $a_{i}(1 \leq i \leq n)$ are nonnegative integers. We define the width of $\omega$, denoted $\operatorname{wd}(\omega)$, to be $\operatorname{wd}(\omega)=\sum a_{i}$.

The problem of determining, for a given Lie algebra, its representation of least dimension $>1$, is quite important and has many applications, notably in problems of maximal subalgebras. (The same could be said for the problem of determining the maximal subgroups of a finite simple group.) Our motivation for studying representations in terms of width arose from a question of Nick Katz. He asked in [10, 22.5] for the simple lie algebras with nonself-dual irreducible representations of dimension $p q$ ( $p, q$ prime). He wanted to know that certain subgroups of classical groups of dimension $p q$ are maximal. He had earlier asked about representations of prime dimension. Using the Weyl dimension formula, Gabber [8, 1.6] showed that the only examples were the natural modules for the Lie algebras $A_{n}$ with $n-1$ prime, $B_{n}$ with $2 n+1$ prime or the seven-dimensional module for $G_{2}$.

Our proof led us to obtain lower bounds for dimensions of irreducible representations whose dominant weight has given width. These results are used in a forthcoming paper by Breuillard and Pisier [3] to bound moments of irreducible representations of Lie groups. This also uses the main result of [6], which gives character estimates. Using our main result then gives good bounds in terms of dimension.

Our first main result determines, among all dominant weights $\omega$ of a given width, the least value of $d=\operatorname{dim} V(\omega)$ and gives an $\omega$ achieving this minimum.

Received by the editors April 1, 2016, and, in revised form, August 11, 2017.

2010 Mathematics Subject Classification. Primary 17B10, 22E46.

Key words and phrases. Complex Lie algebras, representation, highest weight.

The second author was partially supported by NSF grants DMS-1302886 and DMS-1600056. 
Theorem 1.1. Let $\mathfrak{g}$ be a simple Lie algebra of rank $n$. There exists $1 \leq s \leq n$ (depending on $\mathfrak{g})$ such that $\operatorname{dim} V(\omega) \geq \operatorname{dim} V\left(\operatorname{wd}(\omega) \omega_{s}\right)$ for all dominant weights $\omega$.

Equality holds if and only if $\omega=\mathrm{wd}(\omega) \omega_{s^{\prime}}$ and there is an automorphism of the Dynkin digraph of $\mathfrak{g}$ taking $s$ to $s^{\prime}$.

See 4 for a basic reference. We use the Bourbaki notation for Dynkin diagrams, roots, and weights; see [2, Planches II-IX, pp. 250-275]. Let $\Gamma$ be the finite group of graph automorphisms of the Dynkin diagram. Set $m=\min _{1 \leq i \leq n} \operatorname{dim} V\left(\omega_{i}\right)$. The set of $s$ such that $\operatorname{dim} V\left(\omega_{s}\right)=m$ forms a single $\Gamma$-orbit $S$. This orbit has size 3 in the case of $D_{4}(s=1,3,4)$, size 2 in the case of $A_{n}$ and $E_{6}(s=1$ or $n$ ), and size 1 otherwise, in which case $s=n$ if $\mathfrak{g}=F_{4}, E_{7}, E_{8}$ and $s=1$ for $B_{n}, n>2, C_{n}, n>1, D_{n}>4$. (We choose to view $B_{2}$ as $C_{2}$.)

If $\mathfrak{g}$ is classical, then $V\left(t \omega_{s}\right)$ has a nice description in terms of symmetric powers of the natural module. We give the formula for $\operatorname{dim} V\left(t \omega_{s}\right)$ for exceptional $\mathfrak{g}$ in Table 4 of Section 6. Note that, by the Weyl dimension formula, $f_{j}(t)=\operatorname{dim} V\left(t \omega_{j}\right)$ is a polynomial in $t$ of degree the dimension of the nilpotent radical of the corresponding maximal parabolic subalgebra $\mathfrak{p}_{j}$ of $\mathfrak{g}$ (indeed, a positive root $\alpha$ contributes to the product in the numerator if and only if the coefficient of $\alpha_{j}$ in $\alpha$ is nonzero). In particular, letting $t \rightarrow \infty$ in Lemma 2.2 we see the $s \in S$ are precisely those for which the dimension of the nilpotent radical of $\mathfrak{u}_{s}$ of the maximal parabolic subalgebra corresponding to $\alpha_{s}$ is least.

We scale the Killing form $($,$) on \mathfrak{g}^{*} \otimes \mathfrak{g}^{*}$ to be the unique invariant positive definite form such that inner products of the root lattice elements are integral and have $\operatorname{gcd}=1$. Also, by inspection, it follows that $(\omega, 2 \alpha)$ is integral for $\omega$ in the weight lattice and $\alpha$ in the root lattice. Moreover, these inner products are even if the type is not $B$.

Since $\rho=\omega_{1}+\cdots+\omega_{n}$ lies in the weight lattice, the terms in the numerator (and denominator) of the Weyl dimension formula $(\omega+\rho, \alpha)$ (and $(\rho, \alpha))$ are integers if $\mathfrak{g}$ does not have type $B$. The largest term in the numerator of the Weyl dimension formula is $\left(\omega+\rho, \alpha_{h}\right)$, where $\alpha_{h}$ denotes the highest positive root.

Thus, except for type $B, p \leq\left(\omega+\rho, \alpha_{h}\right)$, for any prime divisor $p$ of $\operatorname{dim} V(\omega)$. and, in particular, if $\operatorname{dim} V(\omega)$ is the product of two primes, then

$$
\operatorname{dim} V(\omega) \leq\left(\omega+\rho, \alpha_{h}\right)^{2} .
$$

Our Theorem 1.2 classifies the highest weight representations $V(\omega)$ such that equation (10) holds (even in type B).

Type B will be treated with some care. In type $B$, we have $p \leq(\omega+\rho, 2 \beta)$, where $\beta$ is the highest short root.

Theorem 1.2. Let $\mathfrak{g}$ be a simple Lie algebra of rank $n \geq 2$, and $V(\omega)$ a nontrivial highest weight module module for $\mathfrak{g}$. If $\operatorname{dim} V(\omega) \leq\left(\omega+\rho, \alpha_{h}\right)^{2}$, then $(g, \omega)$ is in Table 1 (if $n>2$ ) or Section 4 (if $n=2$ ).

In particular, we can now easily determine the irreducible modules whose dimension is a product of at most two primes. As noted above, Gabber [8, 1.6] classified those cases where $\operatorname{dim} V(\omega)$ is a prime and our methods give a somewhat different proof of his result. As a consquence, we can now answer the question of Nick Katz [10, 22.5] mentioned above and obtain the following (see Table 5]and [8,12, for more details). 
Corollary 1.3. Let $\mathfrak{g}$ be a simple Lie algebra of rank $n \geq 2$ and $V(\omega)$ an irreducible highest weight $\mathfrak{g}$-module. If $d:=\operatorname{dim} V(\omega)=p q$ with $p$ and $q$ not necessarily distinct primes, then either $\mathfrak{g}$ is classical and $V$ is the natural module or

(1) $d=a(a+2)$ with $a, a+2$ prime;

(2) $d=a(2 a-1)$ with $a, 2 a-1$ prime;

(3) $d=a(2 a+1)$ with $a, 2 a+1$ prime;

(4) $d=a(2 a+3)$ with $a, 2 a+3$ prime;

(5) $d=26,77$ or 133 .

The methods of this paper cannot classify those modules of prime power dimension. If $s$ is any positive integer and $\omega=(s-1) \sum \omega_{i}$, then $\operatorname{dim} V(\omega)=s^{N}$, where $N$ is the number of positive roots for $\mathfrak{g}$ by the homogeneity of the dimension formula as a polynomial in the variables $a_{i}+1$. Taking $s$ to be a prime power gives many such modules.

In some special cases, the possibilities are quite limited. For example, if $\mathfrak{g}$ has rank at least 2 , the only irreducible modules of dimension $p^{2}$ for $p$ an odd prime are the natural module for $\mathfrak{g}$ of type $A_{p^{2}-1}$ or $B_{\left(p^{2}-1\right) / 2}$. Similarly, the only irreducible modules of dimension $2 p$ with $p$ odd are either natural modules or are of dimension $6,10,14$, or 26 . In particular, this gives the following.

Corollary 1.4. Let $\mathfrak{g}=C_{n}$, and let $n$ be an odd prime with $V$ the natural module of dimension $2 n$. If $\mathfrak{h}$ is a proper simple Lie subalgebra of $\mathfrak{g}$ that acts irreducibly on $V$, then $\mathfrak{h}=A_{1}$ or $n=7$ and $\mathfrak{g}=C_{3}$.

Corollary 1.5. Let $\mathfrak{g}$ be a simple Lie algebra of rank $n \geq 2$ and $V(\omega)$ a nontrivial irreducible highest weight $\mathfrak{g}$-module that is not self-dual. If $d:=\operatorname{dim} V(\omega)$ is a product of 2 primes, then $\mathfrak{g}=A_{n}$ and one of the following holds:

(1) $n>3$ and $d=n+1,(n+1)(n+2) / 2$ or $n(n+1) / 2$;

(2) $n=3,4$, or 6 and $d=35$; or

(3) $n=2$ and $d=a(a+1) / 2$ or $a(a+2)$.

In the first case, the modules are (up to duality), the natural module, its symmetric square and its exterior square. The other possibilities can be read off Table 2. As we discuss in Section 6, one can determine whether the module is self-dual and if so what type of form it preserves.

We remark that the same result holds if the characteristic is $\ell>0$ for restricted dominant weights. Let $V(\omega)$ denote the Weyl module with highest weight $\omega$. Then if $\omega$ is restricted and $\operatorname{dim} V(\omega) \leq \ell$, it follows by 7 that this module (which has the same dimension as the corresponding characteristic 0 module) is irreducible.

The paper is organized as follows. In the next section, we give the proof of Theorem 1.1. The proof involves two steps. We first show that if $\omega$ has width $t$, then $\operatorname{dim} V(\omega) \geq \operatorname{dim} V\left(t \omega_{i}\right)$ for some fundamental weight $\omega_{i}$. We then give a case-by-case argument to show the minimum occurs only for the $\omega_{s}$ as described above.

Once we have this result, if $\omega$ has width $t$ and $\operatorname{dim} V\left(t \omega_{s}\right) \leq \operatorname{dim} V(\omega) \leq(\omega+$ $\left.\rho, \alpha_{h}\right)^{2}$, we know that $\operatorname{dim} V\left(t \omega_{s}\right)$ is a polynomial in $t$ of degree the dimension of a nilpotent radical of a maximal parabolic and $\left(\omega+\rho, \alpha_{h}\right)^{2}$ is bounded by a quadratic polynomial in $t$. If $\mathfrak{g}$ has rank at least 2 and $\mathfrak{g}$ is not $A_{2}$, then $\operatorname{dim} V\left(t \omega_{s}\right)$ is a polynomial of degree at least 3 (and typically much larger). Thus, excluding $\mathfrak{g}=A_{2}$, only finitely many $t$ are possible. Indeed, it follows as long the rank of $\mathfrak{g}$ is 
not too small, we will see that $t \leq 2$. In any case, for $t$ fixed, $\operatorname{dim} V(\omega) \leq\left(\omega+\rho, \alpha_{h}\right)^{2}$ will be quadratic in the rank. Moreover, if $\mathfrak{g}$ is classical of large enough rank, it is easy to see that if $\omega$ involves $\omega_{i}$ with $i \geq 3$ (and $i<n-2$ if $\mathfrak{g}$ is of type $A$ ), then $\operatorname{dim} V(\omega)$ will be at least cubic in the rank. Usually one only needs to consider $V\left(a_{1} \omega_{1}+a_{2} \omega_{2}\right)$ with $a_{1}+a_{2}=t \leq 2$.

We make these arguments precise in the following sections and get a complete list of the modules satisfying our required bound. We consider the rank 2 Lie algebras in a separate section. Here one has to work harder and there are more examples (indeed, infinitely many for $A_{2}$ ).

In Section 6] we deduce the corollaries. We also recall some information about the type for self-dual modules and formulas for the minimal dimension of modules of a given width for the exceptional algebras.

Note that some of the results were done by straightforward computations in MAMGA 1 .

\section{Minimal Dimension modules of a Given Width}

Theorem 1.1 follows from Lemmas 2.1 and 2.2 Let $\mathfrak{g}$ be a simple Lie algebra, let $\omega=\sum a_{i} \omega_{i}$ be a dominant weight for $\mathfrak{g}$, where the $\omega_{i}$ are the fundamental dominant weights. We set $t=\sum a_{i}$. Recall that $s$ is chosen so that $\operatorname{dim} V\left(\omega_{s}\right)$ has the smallest dimension among all fundamental dominant weights.

Lemma 2.1. $\operatorname{dim} V(\omega) \geq \min _{1 \leq j \leq n} \operatorname{dim} V\left(t \omega_{j}\right)$.

Lemma 2.2. For all $1 \leq j \leq n, \operatorname{dim} V\left(t \omega_{j}\right) \geq \operatorname{dim} V\left(t \omega_{s}\right)$.

Proof of Lemma 2.1. Suppose that the linear function $m x+b$ takes positive values on the real interval $I$ and set $f(x)=\log (m x+b)$. Then $f^{\prime \prime}(x)=-m^{2} /(m x+b)^{2}<0$, so $f$ is concave downward on $I$.

More generally, the logarithm of a product of linear functions, each of which takes positive values on $I$, is concave downward. Set, as usual, $\langle\gamma, \alpha\rangle=2(\gamma, \alpha) /(\alpha, \alpha)$. Consider the numerator of the Weyl dimension formula

$$
g\left(a_{1}, \ldots, a_{n}\right)=\prod_{\alpha>0}\langle\rho+\omega, \alpha\rangle
$$

where $\omega=a_{1} \omega_{1}+\cdots a_{n} \omega_{n}$. We can view $g$ as a function of the real orthant $a_{1}, \ldots, a_{n} \geq 0$, where it takes values $\geq 1$. In particular, for any real $t \geq 0, g$ takes positive values on the simplex $\Delta_{t}$ consisting of points $P=\left(a_{1}, \ldots, a_{n}\right)$ in the orthant $a_{1}, \ldots, a_{n} \geq 0$ that lie on the hyperplane $\sum a_{i}=t$. Define the support of $P$ to be the number of $a_{i}$ 's that are nonzero.

It is enough to show that for any $P$ in $\Delta_{t}$ with support $\geq 2$, there is a $Q$ in $\Delta_{t}$ with strictly smaller support such that $g(P) \geq g(Q)$.

Assume the support of $P$ is $\geq 2$. Then there are two coordinates of $P$, say $a_{i}$ and $a_{j}$, that are nonzero. Consider the line through $P$ in such that all of the $a_{1}, \ldots, a_{n}$ except $a_{i}$ and $a_{j}$ are fixed, and the sum $a_{i}+a_{j}$ is fixed. Convexity implies that $g$ is minimized on $P \cap \Delta_{t}$ and an endpoint $Q$. Since either $a_{i}$ or $a_{j}=0$ at $Q$, the support of $Q$ is strictly less than the support of $P$. 
Let $\Phi^{+}$be the set of positive roots of $\mathfrak{g}$, and set, as usual, $\rho=\frac{1}{2} \sum_{\alpha \in \Phi^{+}} \alpha=\sum \omega_{i}$. The proof of Lemma 2.2 relies on the Weyl dimension formula [4, Cor. 24.6]:

$$
\operatorname{dim} V(\omega)=\prod_{\alpha \in \Phi^{+}} \frac{\langle\rho+\omega, \alpha\rangle}{\langle\rho, \alpha\rangle} .
$$

We wish to prove that $\operatorname{dim} V\left(t \omega_{j}\right) \geq \operatorname{dim} V\left(t \omega_{s}\right)$. Since, when we apply the Weyl dimension formula, the denominators are the same, it is enough to prove the inequality on the numerators:

$$
\prod_{\alpha \in \Phi^{+}}\left\langle\rho+\omega_{j}, \alpha\right\rangle \geq \prod_{\alpha \in \Phi^{+}}\left\langle\rho+\omega_{s}, \alpha\right\rangle
$$

Note that, for a positive root $\alpha,\left\langle\omega_{i}, \alpha\right\rangle>0$ if and only if $\alpha$ occurs in the unipotent radical, $\mathfrak{u}_{i}$, of the maximal parabolic subalgebra determined by $\alpha_{i}$. In particular, the function $f_{i}(t)=\operatorname{dim} V\left(t \omega_{i}\right)$ is a polynomial in $t$ of degree $\operatorname{dim} \mathfrak{u}_{i}$.

Let $R_{i}$ denote the set of positive roots $\alpha$ which occur in $\mathfrak{u}_{i}$. Our strategy for proving Lemma 2.2 is to find, for each $\mathfrak{g}$ and each $1 \leq j \leq n=\operatorname{rank}(\mathfrak{g})$, an injective function $\phi_{j}$ from $R_{s}$ to $R_{j}$ such that $(1)\langle\rho, \alpha\rangle \leq\left\langle\rho, \phi_{j}(\alpha)\right\rangle$ and $(2)\left\langle\omega_{s}, \alpha\right\rangle \leq$ $\left\langle\omega_{j}, \phi_{j}(\alpha)\right\rangle$ for all $\alpha$ in $R_{s}$. This strategy works in all cases except for $\mathfrak{g}=B_{n}$ and $j=n$ when we do something more elaborate.

Proof of Lemma 2.2. Let $\mathfrak{g}$ be a simple Lie algebra of rank $n$. Let $\omega_{1}, \ldots, \omega_{n}$ be the fundamental dominant weights of $\mathfrak{g}$.

Recall that $\left\langle\rho, \alpha_{j}\right\rangle=1$ and $\left\langle\omega_{i}, \alpha_{j}\right\rangle=\delta_{i, j}$.

We claim that there exists for each $1 \leq j \leq n$ a function $\phi_{j}$ such that (1) and (2) are satisfied. For the finitely many exceptional Lie algebras (types $E, F, G$ ) we verified the claim (and therefore the lemma) by a straightforward computer search using [1]. We proceed to prove the claim case by case for the classical Lie algebras (types $A, B, C, D$ ).

Case $A_{n}, n \geq 2$.

The highest root is $\alpha_{h}=e_{1}-e_{2}$. We have $\left\langle\rho, \alpha_{h}\right\rangle=n-1$. We choose $s=1$ rather than $s=n$. The unipotent radical $u_{1}$ corresponding to $\alpha_{1}$ has dimension $n$. The roots of $\mathfrak{u}_{1}$ are $b_{1}, \ldots, b_{n}$, where $b_{i}=e_{1}-e_{i+1}$. Since all roots have squared-length 2 , we see that $\langle\omega, \alpha\rangle=2(\omega, \alpha) /(\alpha, \alpha)=(\omega, \alpha)$.

Since $\left\langle\omega_{s}, b_{i}\right\rangle=\left(e_{1}, e_{i}-e_{i+1}\right)=1$ for all $b_{i} \in \mathfrak{u}_{1},(2)$ is automatically true. Let $1 \leq j \leq n$. We have $\alpha_{j}=e_{j}-e_{j+1}$ and the roots of $\mathfrak{u}_{j}$ are $\left\{e_{i}-e_{k} \mid 1 \leq i \leq j<\right.$ $k \leq n+1\}$. Set $b_{1}^{\prime}=e_{j-1}-e_{j}, b_{2}^{\prime}=e_{j-2}-e_{j} \cdots, b_{j-1}^{\prime}=b_{j}=e_{1}-e_{j}$ and $b_{i}^{\prime}=b_{i}$ for $i \geq j$. We see that $\phi_{j}\left(b_{i}\right)=b_{i}^{\prime}$ does the trick, since $\left\langle\rho, b_{i}\right\rangle=i=\left\langle\rho, b_{i}^{\prime}\right\rangle$.

Case $C_{n}, n \geq 2$.

Positive Roots $\Phi^{+}=\left\{e_{i} \pm e_{k} \mid 1 \leq i<k \leq n\right\} \cup\left\{2 e_{i} \mid 1 \leq i \leq n\right\}$.

Simple Roots $e_{1}-e_{2}, \ldots, e_{n-1}-e_{n}, 2 e_{n}$.

Fundamental Dominant Weights $e_{1}, e_{1}+e_{2}, \ldots, e_{1}+e_{2}+\cdots+e_{n-1}, e_{1}+e_{2}+\cdots+e_{n}$, $\rho=n e_{1}+(n-1) e_{2}+\cdots+e_{n}$.

We have $s=1$, and $\operatorname{dim} \mathfrak{u}_{1}=2 n-1$. The positive roots in $\mathfrak{u}_{1}$ are $b_{i}=e_{1}-$ $e_{i+1},(1 \leq i \leq n-1), b_{n}=2 e_{i}$, and $b_{i}=e_{1}+e_{2 n+1-i}(n+1 \leq i \leq 2 n-1)$. With this notation, $\left\langle\rho, b_{i}\right\rangle=i$ and $\left\langle\omega_{1}, b_{i}\right\rangle=1$ for all $1 \leq i \leq 2 n-1$, and from the latter property, (2) is automatically true.

$j<n$. The simple root $\alpha_{j}=e_{j}-e_{j+1}$. 
The $j$-th fundamental dominant weight is $\omega_{j}=e_{1}+\cdots+e_{j}$ and the positive roots in $\mathfrak{u}_{j}$ are $\left\{e_{i}-e_{k} \mid 1 \leq i \leq j<k \leq n\right\} \cup\left\{e_{i}+e_{k} \mid 1 \leq i \leq j\right\} \cup\left\{2 e_{i} \mid 1 \leq i \leq j\right\}$.

Set $b_{1}^{\prime}=e_{j}-e_{j+1}, b_{2}^{\prime}=e_{j-1}-e_{j+1}, \ldots, b_{j}^{\prime}=e_{1}-e_{j}$, and set $b_{i}^{\prime}=b_{i}$ for $i>j$. Then $\left\langle\rho, b_{i}^{\prime}\right\rangle=i=\left\langle\rho, b_{i}\right\rangle$ for all $i$, hence (1) is true.

$j=n$. The simple root $2 e_{n}$.

Here, $\omega_{n}=e_{1}+e_{2}+\cdots+e_{n}$. The positive roots in $\mathfrak{u}_{n}$ are $\left\{e_{i}+e_{k} \mid 1 \leq i<k \leq\right.$ $n\} \cup\left\{2 e_{i} \mid 1 \leq i \leq n\right\}$.

Set $b_{1}^{\prime}=2 e_{n}, b_{2}^{\prime}=2 e_{n-1} \cdots b_{n}^{\prime}=2 e_{1}$ and $b_{i}^{\prime}=b_{i}=e_{i}+e_{2 n+1-i}$ for $n+1 \leq i<$ $2 n-1$. One checks that $\left\langle\rho, b_{i}^{\prime}\right\rangle=i$ for all $i$, hence (1) is true.

Case $D_{n}, n \geq 4$

Positive Roots $\Phi^{+}=\left\{e_{i} \pm e_{j} \mid 1 \leq i<j \leq n\right\}$.

Simple Roots $e_{1}-e_{2}, \ldots, e_{n-1}-e_{n}, e_{n-1}+e_{n}$.

Fundamental Dominant Weights $e_{1}, e_{1}+e_{2}, \ldots, e_{1}+e_{2}+\cdots+e_{n-1}, \frac{1}{2}\left(e_{1}+e_{2}+\right.$ $\left.\cdots+e_{n}\right), \frac{1}{2}\left(e_{1}+e_{2}+\cdots+e_{n}\right)$.

We have $\rho=\left(n-\frac{1}{2}\right) e_{1}+\left(n-\frac{3}{2}\right) e_{2}+\cdots \frac{1}{2} e_{n}$. We have $s=1$, and $\operatorname{dim} \mathfrak{u}_{1}=2 n-2$. The roots in $\mathfrak{u}_{1}$ are $b_{i}=e_{1}-e_{i+1}(1 \leq i \leq n-1)$, and $b_{i}=e_{1}+e_{2 n-i}(n \leq i \leq n-2)$. With this notation $\left\langle\rho, b_{i}\right\rangle=i$ and $\left\langle\omega_{1}, b_{i}\right\rangle=1$, so, from the latter, (2) is true. $j<n$. The simple root $e_{j}-e_{j+1}$.

We have $\omega_{j}=e_{1}+\cdots e_{j}$ and the roots in $\mathfrak{u}_{j}$ are $\left\{e_{i}-e_{k} \mid 1 \leq i \leq j<k \leq\right.$ $n\} \cup\left\{e_{i}+e_{k} \mid 1 \leq i \leq j\right\}$. Set $b_{1}^{\prime}=e_{j-1}-e_{j}, b_{2}^{\prime}=e_{j-1}-e_{j}, \ldots, e_{j}=e_{1}-e_{j}$ and $b_{i}^{\prime}=b_{i}$ for $i \geq j$. One checks that $\left\langle\rho{ }^{\prime}{ }_{i}\right\rangle=i$, so (1) is true.

$j=n$. The simple root $e_{n-1}+e_{n}$.

We have $\omega_{n}=\frac{1}{2}\left(e_{1}+\cdots e_{n}\right)$. The roots in $\mathfrak{u}_{n}$ are $\left\{e_{i}+e_{k} \mid 1 \leq i<k \leq n\right\}$. Set $b_{1}^{\prime}=e_{n-2}+e_{n-1}$ (and this is not equal to any other $b_{k}^{\prime}$ since $\left.n \geq 4\right), b_{k}^{\prime}=e_{n-k}+e_{n}$ for $2 \leq k \leq n-1$, and $b_{k}^{\prime}=b_{k}$ if $k \geq n$. One checks that $\left\langle\rho, b_{1}^{\prime}\right\rangle=2>1=\left\langle\rho, b_{1}\right\rangle$ and $\left\langle\rho, b_{i}^{\prime}\right\rangle=i=\left\langle\rho, b_{i}^{\prime}\right\rangle$ for $i>1$ so that (1) is true.

Case $B_{n}, n \geq 3$.

We have $\Phi^{+}=\left\{e_{i} \pm e_{j} \mid 1 \leq i<j \leq n\right\} \cup\left\{e_{i} \mid 1 \leq i \leq n\right\}, \Delta=\left\{e_{1}-e_{2}, e_{2}-\right.$ $\left.e_{3}, \ldots, e_{n-1}-e_{n}, e_{n}\right\}$ and fundamental dominant weights $\omega_{i}=e_{1}+e_{2}+\cdots e_{i}(1 \leq$ $i<n)$ and $\omega_{n}=\frac{1}{2}\left(e_{1}+e_{2}+\cdots e_{n}\right)$, so that $\rho=\left(n-\frac{1}{2}\right) e_{1}+\left(n-\frac{3}{2}\right) e_{2}+\cdots+\frac{1}{2}{ }_{n}$.

We have $s=1$. We have $\operatorname{dim}\left(\mathfrak{u}_{1}\right)=2 n-1$, and the roots in $\mathfrak{u}_{1}$ are $b_{i}=$ $e_{1}-e_{i+1},(1 \leq i \leq n-1), b_{i}=e_{1}+e_{2 n-i}(n \leq i \leq 2 n-2)$ and $b_{2 n-1}=e_{1}$. With this notation, $\left\langle\rho, b_{i}\right\rangle=i$ and $\left\langle\omega_{1}, b_{i}\right\rangle=1$ except for $\left\langle\omega_{1}, b_{2 n-2}\right\rangle=2$.

$j<n$. The simple root $e_{j}-e_{j+1}$.

Set $b_{1}^{\prime}=e_{j-1}-e_{j}, b_{2}^{\prime}=e_{j-2}-e_{j}, \ldots, b_{j-1}^{\prime}=e_{1}-e_{j}$, and set $b_{i}^{\prime}=b_{i}$ for $i \geq j-1$. We have $\left\langle\rho, b_{i}\right\rangle=i=\left\langle\rho, b_{i}^{\prime}\right\rangle$ so that (1) is satisfied. (2) only needs to be checked for the last root, but then $\left\langle\omega_{1}, b_{2 n-1}\right\rangle=2=\left\langle\omega_{j}, b_{2 n-1}^{\prime}\right\rangle$. (This is exactly the same as the argument for $\mathfrak{g}=C_{n}, j<n$.

$j=n$. The simple root $e_{n}$.

The roots in $\mathfrak{u}_{n}$ are $\left\{e_{i}+e_{j} \mid 1 \leq i<j \leq n\right\} \cup\left\{e_{i} \mid 1 \leq i \leq n\right\}$. Set, for the odd subscripts, $b_{1}^{\prime}=e_{n}, b_{3}^{\prime}=e_{n-1}, \ldots, b_{2 n-1}^{\prime}=e_{1}$, and for the even subscripts, $b_{2}^{\prime}=e_{n-1}+e_{n}, b_{4}^{\prime}=e_{n-2}+e_{n-1}, \ldots, b_{2 n-2}^{\prime}=e_{1}+e_{2}$. With this notation, $\left\langle\rho, b_{i}^{\prime}\right\rangle=$ $i$. Both (1) and (2) are true for all roots but the last as $\left\langle\omega_{1}, b_{2 n-1}\right\rangle=2$ whilst $\left\langle\omega_{1}, b_{2 n-1}^{\prime}\right\rangle=1$. Finally, set $b_{2 n-1}^{\prime \prime}=e_{1}+e_{n}$ which is not one of the other $b_{k}^{\prime \prime}$ since $k \geq 3$. One checks, using the following lemma, that the contribution from $b_{2 n-1}$ to the Weyl dimension formula for $V\left(\omega_{1}\right)$ is not greater than the product of 
the contributions to $V\left(\omega_{n}\right)$ from $b_{2 n-1}^{\prime}$ and $b_{2 n-1}^{\prime \prime}$. This finishes the proof of the lemma.

Lemma 2.3. If $t \geq 0$, then

$$
\frac{2 t+(2 n-1)}{2 n-1} \leq \frac{t+(2 n-1)}{2 n-1} \frac{t+n}{n} .
$$

Proof. Take the obvious inequality $n(2 t+2 n-1) \leq(t+n)(t+2 n-1)$ and divide both sides by $2 n-1$.

\section{Classical Lie algebras}

In this section we analyze the four classical types $A, B, C$, and $D$.

3.1. Type A. Let $\mathfrak{g}=\mathfrak{s l}_{n+1}, n>2$ acting on the natural module $V\left(\omega_{1}\right)=\mathbb{C}^{n+1}$. For any integer $t \geq 0$, the module $V\left(t \omega_{1}\right)$ is the $t$-th symmetric power of the natural module. Thus

$$
\operatorname{dim} V\left(t \omega_{1}\right)=\left(\begin{array}{c}
n+t \\
n
\end{array}\right) .
$$

Let $\omega$ be a dominant weight for $\mathfrak{g}$, and set $t=\operatorname{wd}(\omega)$. The highest positive root is $\alpha_{h}=e_{1}-e_{n+1}$, so that $\left(\rho, \alpha_{h}\right)=n$ and $\left(\omega, \alpha_{h}\right)=t$.

We wish to classify all highest weights $\omega$ such that satisfy the inequality

$$
\operatorname{dim} V(\omega) \leq\left(\rho+\omega, \alpha_{h}\right)^{2} .
$$

For such an $\omega$ we have, by Theorem 1.1

$$
\left(\begin{array}{c}
n+t \\
n
\end{array}\right)=\operatorname{dim} V\left(t \omega_{1}\right) \leq \operatorname{dim} V(\omega) \leq\left(\rho+\omega, \alpha_{h}\right)^{2}=(n+t)^{2} .
$$

Assume for the moment that $t \geq 3$. Then $\left(\begin{array}{c}n+t \\ 3\end{array}\right) \leq(n+t)^{2}$, which implies $n+t \leq 8$. Thus $n \leq 5$ and $\operatorname{dim} V(\omega) \leq 64$. An inspection of the Tables 6.6-6.9 of [11] shows that the only possibilities (up to graph automorphism) for $(n, \omega)$ are $\left(n, 3 \omega_{1}\right), 2 \leq n \leq 5,\left(n, 4 \omega_{1}\right), n=2,3$, and $\left(n, 5 \omega_{1}\right), n=2,3$.

For the remainder of the section we assume that $t \leq 2$.

We will use the following result of Lübeck [11, Theorem 5.1] for groups of type A.

Theorem 3.1. Let $\mathfrak{g}=\mathfrak{s l}_{n+1}$ with $n>11$. Assume that $\operatorname{dim} V(\omega) \leq n^{3} / 8$, then (up to a graph automorphism) $\omega=\omega_{1}, 2 \omega_{1} \omega_{2}$ or $\omega_{1}+\omega_{n}$.

One checks that, for any $n, \omega_{1}, 2 \omega_{1}, \omega_{2}$, and $\omega_{1}+\omega_{n}$ all satisfy the inequality.

If $n \leq 11$, Theorem 3.1 implies that $\operatorname{dim} V(\omega) \leq 169$. Inspection of 11, Tables 6.6-6.15] shows that the only additional dominant weights (up to duality) satisfying our inequality are $\left(n, \omega_{3}\right), 2 \leq n \leq 7,\left(3, \omega_{1}+\omega_{2}\right)$, and $\left(3,2 \omega_{2}\right)$.

Now assume that $n>11$. Then $\operatorname{dim} V(\omega) \leq(n+t)^{2}=(n+2)^{2} \leq n^{3} / 8$ and the result now follows by Theorem 3.1 .

3.2. Type B. Let $\mathfrak{g}=B_{n}, n>2$. Let $\mathbb{C}^{2 n+1}=V\left(\omega_{1}\right)$ be the natural module for $\mathfrak{g}$.

We note that in $\operatorname{Sym}^{t}\left(\mathbb{C}^{2 n+1}\right)$, the submodule generated by the multiples, the invariant quadratic form is invariant, and its orthogonal complement is irreducible. In other words, $V\left(t \omega_{1}\right) \oplus \operatorname{Sym}^{t-2}\left(\mathbb{C}^{2 n+1}\right)=\operatorname{Sym}^{t}\left(\mathbb{C}^{2 n+1}\right)$.

Thus, for $t>1, \operatorname{dim} V\left(t \omega_{1}\right)=\left(\begin{array}{c}2 n+t+1 \\ 2 n+1\end{array}\right)-\left(\begin{array}{c}2 n+t-1 \\ 2 n+1\end{array}\right)$.

By Theorem 1.1, we have $\operatorname{dim} V(\omega) \geq \operatorname{dim} V\left(t \omega_{1}\right)$ if $\omega$ has width $t$. 
The highest positive root is $\alpha_{h}=e_{1}+e_{2}$, so that $\left(\rho, \alpha_{h}\right)=2 n-2$ and $\left(\omega, \alpha_{h}\right)=$ $2 t-a_{1}-a_{n} \leq 2 t$, where, as usual, $\omega=\sum a_{i} \omega_{i}$.

Thus $\left(\rho+\omega, \alpha_{h}\right) \leq 2 n+2 t-2$.

Note also that the highest short root is $\beta:=e_{1}$. Note that $(\rho+\omega, 2 \beta) \leq 2 n+2 t-1$. Assume that $\operatorname{dim} V(\omega) \leq\left(\rho+\omega, \alpha_{h}\right)^{2}$. Then for $t>1$,

$$
\left(\begin{array}{c}
2 n+t \\
t
\end{array}\right)-\left(\begin{array}{c}
2 n+t-2 \\
t-2
\end{array}\right) \leq(2 n+2 t-1)^{2} \text {. }
$$

One checks that $\omega_{1}, 2 \omega_{1}, \omega_{2}$, and $\omega_{n}, n \leq 9$ satisfy (10).

We see that the inequality implies $t \leq 3$. In particular, $\operatorname{dim} V(\omega) \leq(2 n+5)^{2}$.

Following Lübeck [11, Theorem 5.1], we have the next Theorem.

Theorem 3.2. Let $\mathfrak{g}$ be a classical Lie algebra of type B, $C$, or $D$ of rank at $n>11$. Assume that $\operatorname{dim} V(\omega) \leq n^{3}$, then $\omega=\omega_{1}, 2 \omega_{1}$ or $\omega_{2}$.

The basic point of the proof is to consider orbits of the Weyl group on the weights.

Thus, for $n>11, \operatorname{dim} V(\omega)<n^{3}$ and so $\omega=\omega_{1}, 2 \omega_{1}$ or $\omega_{2}$. If $n \leq 11$, this is a finite problem and one can check precisely which modules satisfy the inequality (either using the Weyl dimension formula or the tables in [1]. We see that for $n \geq 4$, there are no further examples.

For $n=3$ we see that the only further possibilities are $2 \omega_{3}, 3 \omega_{3}, 3 \omega_{1}$, and $\omega_{1}+\omega_{3}$ (of dimensions $35,112,77$, and 48).

In order to deduce the result about the dimension being a product of two primes, we note that a trivial consequence of the Weyl dimension formula is the following.

Lemma 3.3. Let $\mathfrak{g}$ be of type $B_{n}, n \geq 3$. If $p$ is a prime divisor of $\operatorname{dim} V(\omega)$, then $p \leq(\rho+\omega, 2 \beta)$.

Thus, if $\operatorname{dim} V(\omega)$ is a product of two primes, $\operatorname{dim} V(\omega) \leq(\rho+\omega, 2 \beta)^{2}$. By the classification, we see that it still follows that $\operatorname{dim} V(\omega) \leq\left(\rho+\omega, \alpha_{h}\right)^{2}$.

3.3. Type C. Let $\mathfrak{g}=C_{n}, n>2$. In this case $V\left(\omega_{1}\right)=\mathbb{C}^{2 n}$ is the natural module for $\mathfrak{g}$. It is known that $V\left(t \omega_{1}\right)$ is the $t$-th symmetric power of the natural module.

By Theorem 1.1, if $\omega$ is a dominant weight for $\mathfrak{g}$, then

$$
\operatorname{dim} V(\omega) \geq \operatorname{dim} V\left(t \omega_{1}\right)=\left(\begin{array}{c}
2 n+t-1 \\
2 n-1
\end{array}\right)
$$

where $t=\operatorname{wd}(\omega)$.

Since the highest root for $\mathfrak{g}$ is $\alpha_{h}=2 e_{1}$, we see that $\left(\rho, \alpha_{h}\right)=2 n$ and $\left(\omega, \alpha_{h}\right)=2 t$, so that $\left(\rho+\omega, \alpha_{h}\right)=2 n+2 t$.

Assume that $\omega$ is a dominant weight for $\mathfrak{g}$ that satisfies (11). We see that this implies the inequality

$$
\left(\begin{array}{c}
2 n+t-1 \\
2 n-1
\end{array}\right) \leq(2 n+2 t)^{2} .
$$

We see that $\omega_{1}, \omega_{2}$, and $2 \omega_{1}$ satisfy (1).

For $n \geq 6$, the inequality implies $t \leq 2$, whence $\operatorname{dim} V(\omega) \leq(2 n+4)^{2}$. By Theorem 3.2. this implies that for $n \geq 11$, there are no further examples. For $n<11$, we check directly. We see that in fact for $n \geq 6$, there are no further examples.

If $n \leq 5$, we have the additional possibility $\omega_{3}$. For $n \leq 4$ the inequality implies $t \leq 3$, and one checks that for $n=5$ there are no further possibilities. If $n \leq 4$, 
we have the additional possibilities $\omega_{3}$ and $\omega_{4}$ (if $\left.n=4\right)$. For $n=4$ there are no further possibilities.

Finally, consider $C_{3}$. The inequality implies $t \leq 5$. This leads to the further possibilities $\omega_{1}+\omega_{2}, 3 \omega_{1}$.

3.4. Type D. We consider $G=D_{n}, n \geq 4$ with natural module $\mathbb{C}^{2 n}=V\left(\omega_{1}\right)$. Just as for type $B$, we have $V\left(t \omega_{1}\right) \oplus \operatorname{Sym}^{t-2} V\left(\omega_{1}\right)=\operatorname{Sym}^{t} V\left(\omega_{1}\right)$. It follows that $\operatorname{dim} V\left(t \omega_{1}\right)=\left(\begin{array}{c}2 n+t-1 \\ 2 n-1\end{array}\right)-\left(\begin{array}{c}2 n+t-3 \\ 2 n-1\end{array}\right)$.

The highest positive root is $\alpha_{h}=e_{1}+e_{2}$. If $\omega=\sum a_{i} \omega_{i}$ is a dominant weight, then $\left(\rho, \alpha_{h}\right)=2 n-3 \leq 2 n+2 t-3 . \quad\left(\omega, \alpha_{h}\right)=2 t-\left(a_{1}+a_{n-1}+a_{n}\right)$ so that $\left(\omega+\rho, \alpha_{h}\right)=2 n-3+2 t-\left(a_{1}+a_{n-1}+a_{n}\right) \leq 2 n+2 t-3$.

Assume now that (10) is true. Then we have the inequality

$$
\left(\begin{array}{c}
2 n+t-1 \\
2 n-1
\end{array}\right)-\left(\begin{array}{c}
2 n+t-3 \\
2 n-1
\end{array}\right) \leq(2 n+2 t-3)^{2} .
$$

One checks that $\omega_{1}, 2 \omega_{1}$, and $\omega_{2}$ satisfy (11). One also checks that the half-spin representation $\omega_{n}$ works for $n \leq 9$.

If $n \geq 5$, the inequality implies $t \leq 2$, whence $\operatorname{dim} V(\omega) \leq(2 n+1)^{2}$. As in the previous cases, this implies by Theorem 3.2 that for $n \geq 11$, there are no further examples. One checks for $5 \leq n \leq 10$, there are no other examples as well.

If $n=4$, the inequality implies $t \leq 4$. A computer check finds no further examples (up to the (large) group of graph automorphisms).

\section{RANK 2 LiE ALGEBRAS}

Let $\mathfrak{g}$ be a rank 2 Lie algebra. Let $\omega=a \omega_{1}+b \omega_{2}$ be a dominant weight for $\mathfrak{g}$, where $\omega_{1}$ and $\omega_{2}$ are fundamental dominant weights for $\mathfrak{g}$ and $a, b \geq 0$. If $\operatorname{dim} V(\omega)$ is a product of two (not necessarily distinct) primes, then necessarily

$$
\operatorname{dim} V(\omega) \leq\left(\rho+\omega, \alpha_{h}\right)^{2} .
$$

We also find all dominant weights such that the inequality holds, and among them we identify those such that $\operatorname{dim} V(\omega)=p q$.

4.1. $A_{2}$. The inequality to be solved is $(a+1)(b+1)(a+b+2) / 2 \leq(a+b+2)^{2}$. This is equivalent to $(a+1)(b+1) \leq 2(a+b+2)$, or $(a-1)(b-1) \leq 4$. The solutions are: $a=0, b$ any, $a=1, b$ any, $b=0, a$ any, $b=1, a$ any, $a=2, b \leq 4, b=2, a \leq 4$, or $a=b=3$. The cases where $\operatorname{dim} V(\omega)=p q$ are (up to interchanging $a$ and $b$ ): If $u$ and $2 u+1$ are prime: $\operatorname{dim} V\left((2 u-1) \omega_{1}\right)=u(2 u+1)$. If $u$ and $2 u-1$ are prime: $\operatorname{dim} V\left((2 u-2) \omega_{1}\right)=u(2 u-1)$. If $u$ and $u+2$ are prime: $\operatorname{dim} V\left((u-1) \omega_{1}+\omega_{2}\right)=$ $u(u+2)$.

4.2. $C_{2}$. Let $\omega=a \omega_{1}+b \omega_{2}$ be a dominant weight for $\mathfrak{g}=C_{2}$. Then $\operatorname{dim} V(\omega)=$ $(a+1)(2 b+2)(a+2 b+3)(2 a+2 b+4) / 24$ and $\left(\rho+\omega, \alpha_{h}\right)=(2 a+2 b+4)$. The inequality to be solved, $(a+1)(2 b+2)(a+2 b+3)(2 a+2 b+4) / 24 \leq(2 a+2 b+4)^{2}$, is equivalent to

$$
(a+1)(b+1)(a+2 b) \leq 24(a+b+2) .
$$


This has 53 solutions:

$$
\begin{aligned}
& a=0 \text { and } \quad b \leq 11, \\
& a=1 \text { and } \quad b \leq 5, \\
& a=2 \text { and } \quad b \leq 3, \\
& b=0 \text { and } \quad a \leq 22, \\
& b=1 \text { and } \quad a \leq 9, \\
& b=2 \text { and } \quad a \leq 5, \\
& a=3 \text { and } \quad b=3 .
\end{aligned}
$$

Of these, 7 have dimension $p q$, namely: $\operatorname{dim} V\left(\omega_{1}\right)=4, \operatorname{dim} V\left(2 \omega_{1}\right)=10, \operatorname{dim} V\left(2 \omega_{2}\right)$ $=14, \operatorname{dim} V\left(2 \omega_{1}+\omega_{2}\right)=\operatorname{dim} V\left(4 \omega_{1}\right)=35, \operatorname{dim} V\left(4 \omega_{2}\right)=55$, and $\operatorname{dim} V\left(5 \omega_{2}\right)=91$.

4.3. $G_{2}$. Let $\omega=a \omega_{1}+b \omega_{2}$ be a dominant weight for $\mathfrak{g}=G_{2}$. Then $\operatorname{dim} V(\omega)=$ $(a+1)(b+1)(a+b+2)(a+2 b+3)(a+3 b+4)(2 a+3 b+5) / 120$, and $\left(\rho+\omega, \alpha_{h}\right)=$ $2 a+3 b+5$. The inequality to be solved is $(a+1)(b+1)(a+b+2)(a+2 b+3)(a+3 b+4) \leq$ $120(2 a+3 b+5)$.

We claim that the inequality implies $a, b \leq 7$. If $b \geq a$, then the left hand side is at least $(a+1)^{4}$, and the right hand side is at most $120 \cdot 5(a+1)$, whence $b \leq a \leq 7$. There is a similar argument assuming $a \leq b$.

One checks that the inequality has the 7 solutions

$$
(a, b)=(0,0),(0,1),(0,2),(1,0),(1,1),(1,2),(1,3) .
$$

Of these, 3 have dimension $p q$, namely: $\operatorname{dim} V\left(\omega_{2}\right)=14$, and $\operatorname{dim} V\left(2 \omega_{2}\right)=$ $\operatorname{dim} V\left(3 \omega_{1}\right)=77$.

\section{Exceptional Lie algebras}

5.1. $F_{4}$. In this section, we prove the following.

Proposition 5.1. Let $\mathfrak{g}=F_{4}$. If $\omega \neq 0$ and $\operatorname{dim} V(\omega) \leq\left(\rho+\omega, \alpha_{h}\right)^{2}$, then $V(\omega)$ is the either the minimal module of dimension 26 or the adjoint module of dimension 52 .

Assume that $\omega=\sum a_{i} \omega_{i}$ with $t=\sum a_{i}$ and $\operatorname{dim} V(\omega) \leq\left(\rho+\omega, \alpha_{h}\right)^{2}$. Note that $\left(\rho+\omega, \alpha_{h}\right)=\left(2 a_{1}+3 a_{2}+4 a_{3}+2 a_{4}+16\right) \leq 4 t+11$.

Let $f(t)$ be the function given in Table 2 for $F_{4}$. Then $\operatorname{dim} V(\omega) \leq f(t) / f(0)$. It is straightforward to see that this implies that $t \leq 2$, whence $\operatorname{dim} V(\omega) \leq 19^{2}$. The only additional $\omega$ that satisfy this bound are $\omega_{3}$ and $2 \omega_{4}$ (of dimensions 273 and 324 , respectively). These do not satisfy the required inequality, whence the result.

5.2. $E_{n}, n=6,7,8$. First consider $\mathfrak{g}=E_{6}$.

Proposition 5.2. Let $\mathfrak{g}=E_{6}$. If $\omega \neq 0$ and $\operatorname{dim} V(\omega) \leq\left(\rho+\omega, \alpha_{h}\right)^{2}$, then $V(\omega)$ is either one the two minimal modules of dimension 27 or the adjoint module of dimension 78 .

Proof. Let $\omega=\sum a_{i} \omega_{i}$ with $t=\sum a_{i}$. Let $f(t)$ be the polynomial given in Table 4 for $E_{6}$. Thus, $\operatorname{dim} V(\omega) \leq f(t) / f(0)$. Note that $\left(\rho+\omega, \alpha_{h}\right)=a_{1}+2 a_{2}+2 a_{3}+3 a_{4}+$ $2 a_{5}+a_{6}+11 \leq 5 t+11$. It is straightforward to see that this forces $t \leq 2$, and in particular, $\operatorname{dim} V(\omega) \leq 21^{2}$. Inspection of the tables in [11] implies the result.

The same proof (only easier since the polynomial $f(t)$ has larger degree) gives the following for $E_{7}$ and $E_{8}$. 
Proposition 5.3. Let $\mathfrak{g}=E_{7}$. If $\omega \neq 0$ and $\operatorname{dim} V(\omega) \leq\left(\rho+\omega, \alpha_{h}\right)^{2}$, then $V(\omega)$ is either the minimal module of dimension 56 or the adjoint module of dimension 133 .

Proposition 5.4. Let $\mathfrak{g}=E_{8}$. If $\omega \neq 0$ and $\operatorname{dim} V(\omega) \leq\left(\rho+\omega, \alpha_{h}\right)^{2}$, then $V(\omega)$ is the adjoint module of dimension 248.

\section{The COROLlaRies}

It follows by the Weyl dimension formula that aside from type $B$, the largest prime dividing the dimension of $\operatorname{dim} V(\omega)$ is at most $\left(\omega+\rho, \alpha_{h}\right)$. Thus, if $\operatorname{dim} V(\omega)$ is a product of at most 2 primes, it follows that $\operatorname{dim} V(\omega) \leq\left(\omega+\rho, \alpha_{h}\right)^{2}$.

Thus, Corollaries 1.3, 1.4 and 1.5 follow immediately from Theorem 1.2 except for type $B$.

If $\mathfrak{g}$ has type $B$, then the Weyl dimension formula implies that the largest prime dividing the dimension of $\operatorname{dim} V(\omega)$ is at most $(\omega+\rho, \beta)$, where $\beta$ is the highest short root. Thus, if $\operatorname{dim} V(\omega)$ is a product of at most 2 primes, $\operatorname{dim} V(\omega) \leq(\omega+\rho, \beta)^{2}$. In Section 3, Subection 3.2 we showed that this implied that $\operatorname{dim} V(\omega) \leq\left(\omega+\rho, \alpha_{h}\right)^{2}$ and so again the corollaries follow from Theorem 1.2

We summarize in Table 2 when the irreducible highest weight module $V(\omega)$ is self-dual, and, in the positive case, whether the module is symplectic or orthogonal. For convenience, define $2 k+1$ to be the largest odd integer $\leq n$. The first column of Table 2 lists the simple Lie algebra, the second column gives necessary and sufficient conditions for $V(\omega)$ to be self-dual, the third gives necessary and sufficient conditions for the self-dual representation $V(\omega)$ to be symplectic. Table 3 gives the fundamental dominant weight modules that are symplectic.

It is easy to tell whether an irreducible module is self-dual. All modules are selfdual if there is no graph automorphism or for type $D_{n}$ with $n$ even. In the other cases, $V(\omega)$ is self-dual if and only if it is invariant under the graph automorphism, where $\omega=\sum_{i=1}^{n} a_{i} \omega_{i}$ is a dominant weight.

If a module is self-dual, then it either supports a nonzero invariant alternating form (symplectic case) or a nonzero invariant symmetric form (orthogonal case) but not both. See, for example, [5, 5.1.24] for the well-known criterion to distinguish these cases. To distinguish the orthogonal from the symplectic cases, it is sufficient to do so for the fundamental dominant weights. Indeed, let $B$ denote the set of fundamental dominant weights $\omega_{i}$ such that $V\left(\omega_{i}\right)$ is (self-dual and) symplectic. Then the self-dual highest weight module $V(\omega)$ is symplectic if and only if $\sum_{i \in B} a_{i}$ is odd.

We list all symplectic fundamental dominant weights. 
TABLE 1. Nonzero $\omega$ such that $\operatorname{dim} V(\omega) \leq\left(\rho+\omega, \alpha_{h}\right)^{2}$ and rank $n \geq 3$ (up to graph automorphism).

\begin{tabular}{|c|c|c|}
\hline $\mathfrak{g}$ & $\omega$ & $\operatorname{dim} V(\omega)$ \\
\hline \multirow[t]{4}{*}{$\overline{A_{n}}$} & $\omega_{1}$ & $n+1$ \\
\hline & $2 \omega_{1}$ & $(n+1)(n+2) / 2$ \\
\hline & $\omega_{2}$ & $n(n+1) / 2$ \\
\hline & $\omega_{1}+\omega_{n}$ & $n(n+2)$ \\
\hline \multirow{5}{*}{$\begin{array}{l}3 \leq n \leq 7 \\
3 \leq n \leq 5 \\
A_{3}\end{array}$} & $\omega_{3}$ & $\left(\begin{array}{c}n+1 \\
3\end{array}\right)$ \\
\hline & $3 \omega_{1}$ & $\left(\begin{array}{c}n+3 \\
3\end{array}\right)$ \\
\hline & $t \omega_{1}, t=4,5$ & 35,56 \\
\hline & $\omega_{1}+\omega_{2}$ & 20 \\
\hline & $2 \omega_{2}$ & 45 \\
\hline \multirow[t]{3}{*}{$B_{n}$} & $\omega_{1}$ & $2 n+1$ \\
\hline & $2 \omega_{1}$ & $n(2 n+3)$ \\
\hline & $\omega_{2}$ & $n(2 n+1)$ \\
\hline \multirow{5}{*}{$\begin{array}{l}3 \leq n \leq 9 \\
B_{3}\end{array}$} & $\omega_{n}$ & $2^{n}$ \\
\hline & $2 \omega_{3}$ & 35 \\
\hline & $\omega_{1}+\omega_{3}$ & 48 \\
\hline & $3 \omega_{1}$ & 77 \\
\hline & $3 \omega_{3}$ & 112 \\
\hline \multirow[t]{3}{*}{$C_{n}$} & $\omega_{1}$ & $2 n$ \\
\hline & $2 \omega_{1}$ & $n(2 n+1)$ \\
\hline & $\omega_{2}$ & $(n-1)(2 n+1)$ \\
\hline \multirow[t]{2}{*}{$3 \leq n \leq 5$} & $3 \omega_{1}$ & $\left(\begin{array}{c}2 n+2 \\
3\end{array}\right)$ \\
\hline & $\omega_{3}$ & $\left(\begin{array}{c}2 n \\
3\end{array}\right)-2 n$ \\
\hline$C_{4}$ & $\omega_{4}$ & 42 \\
\hline$C_{3}$ & $\omega_{1}+\omega_{2}$ & 64 \\
\hline \multirow[t]{3}{*}{$D_{n}(4 \leq n)$} & $\omega_{1}$ & $2 n$ \\
\hline & $2 \omega_{1}$ & $(2 n-1)(n-1)$ \\
\hline & $\omega_{2}$ & $n(2 n-1)$ \\
\hline $4 \leq n \leq 9$ & $\omega_{n}$ & $2^{n-1}$ \\
\hline \multirow[t]{2}{*}{$E_{6}$} & $\overline{\omega_{1}}$ & 27 \\
\hline & $\omega_{2}$ & 78 \\
\hline \multirow[t]{2}{*}{$E_{7}$} & $\omega_{7}$ & 56 \\
\hline & $\omega_{1}$ & 133 \\
\hline$E_{8}$ & $\omega_{8}$ & 248 \\
\hline \multirow[t]{2}{*}{$F_{4}$} & $\omega_{4}$ & 26 \\
\hline & $\omega_{1}$ & 52 \\
\hline
\end{tabular}


TABLE 2. Duality.

\begin{tabular}{|l|c|c|}
\hline $\mathfrak{g}$ & $V(\omega)$ Self - Dual? & Symplectic? \\
\hline \hline$A_{n}, n \geq 1$ & For all $1 \leq \mathrm{i} \leq \mathrm{n}, \mathrm{a}_{\mathrm{i}}=\mathrm{a}_{\mathrm{n}+1-\mathrm{i}}$ & $n \equiv 1$ mod 4 and $\mathrm{a}_{\mathrm{k}+1}$ odd \\
$B_{n}, n \geq 3$ & always & $n \equiv 1$ or 2 mod 4 and $\mathrm{a}_{\mathrm{n}}$ odd \\
$C_{n}, n \geq 2$ & always & $a_{1}+a_{3}+\cdots+a_{2 k+1}$ odd \\
$D_{n}, n \geq 4$ even & always & $n \equiv 2$ mod 4 and $\mathrm{a}_{\mathrm{n}-1}+\mathrm{a}_{\mathrm{n}}$ odd \\
$D_{n}, n \geq 5$ odd & $a_{n-1}=a_{n}$ & never \\
$E_{6}$ & $a_{1}=a_{6}$ and $\mathrm{a}_{2}=\mathrm{a}_{5}$ & never \\
$E_{7}$ & always & $a_{2}+a_{5}+a_{7}$ odd \\
$E_{8}$ & always & never \\
$G_{2}$ & always & never \\
$F_{4}$ & always & never \\
\hline
\end{tabular}

TABLE 3. Fundamental dominant weight modules $V\left(\omega_{i}\right)$ that are symplectic.

\begin{tabular}{|l|c|}
\hline $\mathfrak{g}$ & $i$ \\
\hline \hline$A_{n}, n \equiv 1 \quad \bmod 4$ & $(n+1) / 2$ \\
\hline$B_{n}, n \equiv 1,2 \quad \bmod 4$ & $n$ \\
\hline$C_{n}$ & $i$ odd \\
\hline$D_{n}, n \equiv 2 \quad \bmod 4$ & $n-1, n$ \\
\hline$E_{7}$ & $2,5,7$ \\
\hline
\end{tabular}

TABLE 4. This table gives a monic polynomial $f(t)$ such that $\operatorname{dim} V\left(t \omega_{s}\right)=f(t) / f(0)$ for $\mathfrak{g}$ exceptional. Note that $\operatorname{dim} V\left(t \omega_{s}\right)$ is the least dimension among all highest weight modules $V(\omega)$ of width $\operatorname{wd}(\omega)=t$.

\begin{tabular}{|l|c|c|}
\hline $\mathfrak{g}$ & $f(t)$ & degree(f) \\
\hline$G_{2}$ & $(t+1)(t+2)(t+3)(t+4)(2 t+5)$ & 5 \\
$F_{4}$ & $\prod_{1}^{10}(t+j) \prod_{4}^{7}(t+j) \cdot(2 t+11)$ & 15 \\
$E_{6}$ & $\prod_{1}^{11}(t+j) \prod_{4}^{8}(t+j)$ & 16 \\
$E_{7}$ & $\prod_{1}^{17}(t+j) \prod_{5}^{13}(t+9)$ & 27 \\
$E_{8}$ & $\prod_{1}^{28}(t+j) \prod_{6}^{23}(t+j) \prod_{10}^{19}(t+j) \cdot(2 t+29)$ & 57 \\
\hline
\end{tabular}


TABLE 5. $\operatorname{dim} V(\omega)=p q$ with $p$ and $q$ prime.

\begin{tabular}{|c|c|c|c|}
\hline$p q$ & $\mathfrak{g}$ & $\omega$ & Duality \\
\hline \multirow[t]{5}{*}{ any } & $\overline{A_{1}}$ & $(p q-1) \omega_{1}$ & 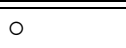 \\
\hline & $A_{p q-1}$ & $\omega_{1}$ & - \\
\hline & $C_{p q / 2}(p q$ even $)$ & $\omega_{1}$ & - \\
\hline & $B_{(p q-1) / 2}(p q$ odd $)$ & $\omega_{1}$ & + \\
\hline & $D_{p q / 2}(p q$ even $)$ & $\omega_{1}$ & + \\
\hline \multirow[t]{6}{*}{$a(2 a+1)$} & $A_{2 a-1}$ & $2 \omega_{1}$ & o \\
\hline & $A_{2 a}$ & $\omega_{2}$ & ० \\
\hline & $B_{a}(a>2)$ & $\omega_{2}$ & + \\
\hline & $C_{a}$ & $2 \omega_{1}$ & + \\
\hline & $A_{2}$ & $(2 a-1) \omega_{1}$ & $\circ$ \\
\hline & $D_{a+1}$ & $2 \omega_{1}$ & + \\
\hline \multirow[t]{5}{*}{$a(2 a-1)$} & $A_{2 a-2}$ & $2 \omega_{1}$ & o \\
\hline & $A_{2 a-1}(a>2)$ & $\omega_{2}$ & ○ \\
\hline & $A_{3}$ & & + \\
\hline & $A_{2}$ & $(2 a-2) \omega_{1}$ & $\circ$ \\
\hline & $D_{a}$ & $\omega_{2}$ & + \\
\hline$a(a+2)$ & $\begin{array}{l}A_{a} \\
A_{2}\end{array}$ & $\begin{array}{l}\omega_{1}+\omega_{n} \\
(a-1) \omega_{1}+\omega_{2}\end{array}$ & $\begin{array}{l}+ \\
\end{array}$ \\
\hline \multirow[t]{2}{*}{$a(2 a+3)$} & $B_{a}$ & $2 \omega_{1}$ & + \\
\hline & $C_{a+1}$ & $\omega_{2}$ & + \\
\hline \multirow[t]{3}{*}{14} & $C_{2}$ & $2 \omega_{2}$ & + \\
\hline & $C_{3}$ & $\omega_{3}$ & - \\
\hline & $G_{2}$ & $\omega_{2}$ & + \\
\hline 26 & $F_{4}$ & $\omega_{4}$ & + \\
\hline \multirow[t]{6}{*}{35} & $A_{3}$ & $4 \omega_{1}$ & o \\
\hline & $A_{4}$ & $3 \omega_{1}$ & ० \\
\hline & $A_{6}$ & $\omega_{3}$ & ○ \\
\hline & $B_{3}$ & $2 \omega_{3}$ & + \\
\hline & $C_{2}$ & $2 \omega_{1}+\omega_{2}$ & + \\
\hline & $C_{2}$ & $4 \omega_{1}$ & + \\
\hline 55 & $C_{2}$ & $4 \omega_{2}$ & + \\
\hline \multirow[t]{3}{*}{77} & $B_{3}$ & $3 \omega_{1}$ & + \\
\hline & $G_{2}$ & $2 \omega_{2}$ & + \\
\hline & $G_{2}$ & $3 \omega_{1}$ & + \\
\hline 91 & $C_{2}$ & $5 \omega_{2}$ & + \\
\hline 133 & $E_{7}$ & $\omega_{1}$ & + \\
\hline
\end{tabular}




\section{ACKNOWLEDGMENTS}

The authors thank Nick Katz for asking the question about representations with dimension a product of two primes.

\section{REFERENCES}

[1] W. Bosma, J. Cannon, and C. Playoust, The Magma algebra system. I. The user language, J. Symbolic Comput. 24 (1997), no. 3-4, 235-265, DOI 10.1006/jsco.1996.0125. Computational algebra and number theory (London, 1993). MR.1484478

[2] N. Bourbaki, Éléments de mathématique. Fasc. XXXIV. Groupes et algèbres de Lie. Chapitre IV: Groupes de Coxeter et systèmes de Tits. Chapitre V: Groupes engendrés par des réflexions. Chapitre VI: systèmes de racines (French), Actualités Scientifiques et Industrielles, No. 1337, Hermann, Paris, 1968. MR0240238

[3] E. Breuillard and G. Pisier, Remarks on random unitaries and amenable linear groups, in preparation.

[4] W. Fulton and J. Harris, Representation theory: A first course; Readings in Mathematics, Graduate Texts in Mathematics, vol. 129, Springer-Verlag, New York, 1991. MR1153249

[5] R. Goodman and N. R. Wallach, Representations and invariants of the classical groups, Encyclopedia of Mathematics and its Applications, vol. 68, Cambridge University Press, Cambridge, 1998. MR1606831

[6] K. E. Hare, The size of characters of compact Lie groups, Studia Math. 129 (1998), no. 1, 1-18, DOI 10.4064/sm-129-1-1-18. MR1611918

[7] J. C. Jantzen, Low-dimensional representations of reductive groups are semisimple, Algebraic groups and Lie groups, Austral. Math. Soc. Lect. Ser., vol. 9, Cambridge Univ. Press, Cambridge, 1997, pp. 255-266. MR 1635685

[8] N. M. Katz, Exponential sums and differential equations, Annals of Mathematics Studies, vol. 124, Princeton University Press, Princeton, NJ, 1990. MR.1081536

[9] N. M. Katz, Gauss sums, Kloosterman sums, and monodromy groups, Annals of Mathematics Studies, vol. 116, Princeton University Press, Princeton, NJ, 1988. MR.955052

[10] N. M. Katz, Convolution and equidistribution: Sato-Tate theorems for finite-field Mellin transforms, Annals of Mathematics Studies, vol. 180, Princeton University Press, Princeton, NJ, 2012. MR2850079

[11] F. Lübeck, Small degree representations of finite Chevalley groups in defining characteristic, LMS J. Comput. Math. 4 (2001), 135-169, DOI 10.1112/S1461157000000838. MR1901354

[12] R. Steinberg, Lectures on Chevalley groups, Yale University, New Haven, Conn., 1968. Notes prepared by John Faulkner and Robert Wilson. MR0466335

Center for Communications Research, San Diego, California 92121

Email address: danielgolds@gmail.com

Department of Mathematics, University of Southern California, Los Angeles, CALIFORNIA 90089-2532

Email address: guralnic@usc.edu

Center for Communications Research, San Diego, California 92121

Email address: stong@ccrwest.org 